\title{
Qualidade de vida de mulheres e homens idosos em situação de violência por parceiro íntimo
}

\author{
Quality of life of older women and men in situations of intimate partner violence
}

Deise Warmling' (ID

Carolina Abreu Henn de Araújo' (DD

Sheila Rubia Lindner' (1D

Elza Berger Salema Coelho' $\mathbb{D}$

\section{Resumo}

Este artigo buscou investigar a associação entre a violência por parceiro íntimo (VPI) e os níveis de qualidade de vida (QV) e seus domínios (controle e autonomia; realização pessoal e prazer), em pessoas idosas. Realizou-se estudo transversal de base populacional da segunda onda (2013/2014) do Estudo EpiFloripa, com pessoas idosas (n=649), residentes em Florianópolis, SC, Sul do Brasil. As médias de QV e seus domínios foram descritas segundo as variáveis de ajuste (idade, renda, deficit cognitivo, sintomas depressivos e dependência de atividades de vida diária), estratificadas por sexo. Analisou-se a associação entre VPI e QV por meio de regressão linear múltipla, com significância estatística de $5 \%$. Os escores médios de QV, bem como realização pessoal e prazer foram semelhantes entre os sexos, enquanto o controle e autonomia das mulheres foram significativamente inferiores em comparação aos homens $(p=0,04)$. Observou- se menores escores de QV nas mulheres expostas à situação de violência, nas três direcionalidades analisadas: perpetrada (-3,15; IC95\%: -4,84; -1,45), bidirecional (-2,59; IC95\%: -4,10; -1,09) e sofrida $(-1,62 ;$ IC95\%: $-3,06 ;-0,17)$ e as mais prejudicadas foram aquelas que cometeram agressões. O controle e autonomia foi afetado para as perpetradoras e envolvidas na violência bidirecional, enquanto menores escores de realização pessoal e prazer foram verificados entre as idosas, em quaisquer situações de violência. Os homens não tiveram sua QV prejudicada por estarem em situação de VPI, tanto vítimas como perpetradores dessa violência. Concluiu-se que a VPI possui impacto assimétrico sobre a QV de pessoas idosas em relação ao sexo, prejudicando com maior intensidade as mulheres.

\footnotetext{
Universidade Federal de Santa Catarina, Departamento de Saúde Pública, Programa de Pós-Graduação em Saúde Coletiva. Florianópolis, SC, Brasil.
}

Não houve financiamento para a execução desse trabalho.

Os autores declaram não haver conflito na concepção deste trabalho.

Palavras-chaves: Violência por Parceiro Íntimo. Qualidade de Vida. Homens. Mulheres. Saúde do Idoso. 


\section{Abstract}

The present paper sought to investigate the association between intimate partner violence (IPV) and the levels of quality of life (QoL) and its domains (control and autonomy; personal fulfillment and pleasure), in older people. A population-based cross-sectional study of the second wave (2013/2014) of the EpiFloripa Study was carried out with older people $(\mathrm{n}=649)$ living in Florianópolis, SC, Southern Brazil. The average QoL score and their domains were described according to the adjustment variables (age, income, cognitive deficit, depressive symptoms, and dependence on activities of daily living) stratified by gender. The relation between IPV and QoL was analyzed using multiple linear regression with a statistical significance of $5 \%$. The average scores of QoL, personal fulfillment, and pleasure were similar between genders, while control and women's autonomy were significantly lower compared to men $(p=0.04)$. Lower QoL scores were observed in women exposed to violence in the three directionalities analyzed: perpetrated (-3.15; 95\%CI: $-4.84 ;-1.45)$, bidirectional (-2.59; 95\%CI: -4.10; $-1.09)$, and suffered $(-1,62 ; 95 \% \mathrm{CI}:-3.06 ;-0.17)$; the most affected ones were those who were aggressive. The control and autonomy were affected for the perpetrators and those involved in bidirectional violence, while lower scores of personal fulfillment and pleasure were seen among the older women in any violent situation. Men did not have their QoL impaired due to IPV, neither as victims nor perpetrators of this violence. It was concluded that IPV has an asymmetric impact on the QoL of older people when it comes to gender with women being the most affected ones.

\section{INTRODUÇÃo}

Com o crescimento acentuado do envelhecimento populacional, torna-se fundamental assegurar que pessoas idosas possam vivenciar essa fase enquanto experiência positiva e com qualidade de vida. Para isso, é essencial que estejam livres de quaisquer formas de violência, em condições seguras e dignas de vida ${ }^{1}$. A violência por parceiro íntimo (VPI) define-se por qualquer ato de violência, física, sexual, psicológica ou abuso econômico ${ }^{2}$.

A exposição à violência pode resultar em sofrimento desnecessário, lesão ou dor, perda ou violação dos direitos humanos, transtorno de estresse pós-traumático e somatização, tendendo a afetar severamente a saúde física e mental das vítimas, fatores que influenciam em menores níveis de qualidade de vida $(\mathrm{QV})^{3}$. Para pessoas idosas, a QV é definida pela satisfação em quatro domínios: controle, relativo à capacidade de intervir ativamente em seu ambiente; autonomia, relacionada ao direito de estar livre da interferência indesejada dos outros; prazer, que se refere à busca por atividades agradáveis; e por fim, a realização pessoal que descreve o desenvolvimento pleno de si mesmo ${ }^{4,5}$.
Para o alcance da QV, ressaltam-se as relações sociais, inclusive o bem-estar no relacionamento íntimo. Porém, embora ainda oculta entre pessoas idosas, a VPI se manifesta como uma forma de comunicação e pode se estabelecer como alternativa para resolução de conflitos $^{6}$, que muitas vezes, inicia na vida adulta.

A VPI consiste em uma violação dos direitos humanos, com grande magnitude na população mundial ${ }^{7}$. Estudos estimam que mais de $30 \%$ das mulheres e cerca de $25 \%$ dos homens sofram esse tipo de violência em todo o mundo ${ }^{8}$, embora a vitimização nos homens seja menos investigada e explorada na literatura. Ao verificar a prevalência de VPI física, foi constatado que mais homens $(6,4 \%)$ em comparação às mulheres $(5,0 \%)$ sofreram tais agressões na Dinamarca ${ }^{9}$. Esse achado dá visibilidade às agressões praticadas contra homens, transcende a unidirecionalidade da violência e reforça a necessidade de investigar ambos os sexos, como possíveis vítimas ou agressores no relacionamento íntimo.

Ao analisar o efeito da violência física ${ }^{9}$, psicológica e comportamento controlador em parceiros íntimos verificou-se perda da QV em mulheres idosas. Estudo dinamarquês que explorou a associação de VPI e
Keywords: Intimate Part
Violence. Quality of life. Men. Women. Health of the Elderly. 
QV em diversas faixas etárias, concluiu que pessoas idosas tiveram maior redução de QV quando expostas à violência cometida pelo parceiro, comparadas às adultas, ressaltando a relevância de investigar o fenômeno também nessa faixa etária.

A investigação do efeito da VPI sobre a QV em pessoas idosas, embora ainda escassa na literatura, vem aos poucos sendo explorada em estudos europeus $^{3,9}$ e norte-americanos ${ }^{10}$ onde o contexto de envelhecimento é mais presente. Entretanto, nenhum dos estudos analisou o impacto da VPI sobre a QV e seus domínios, segundo a direcionalidade da violência e sexo das pessoas idosas. No Brasil, não foram encontradas publicações com a população idosa referente ao tema, explicitando a lacuna no conhecimento e o ineditismo deste estudo. Diante deste contexto, busca-se investigar a associação entre a VPI (sofrida, perpetrada e bidirecional) e os níveis de QV em seus domínios (controle e autonomia; realização pessoal e prazer) em mulheres e homens idosos residentes em Florianópolis, Santa Catarina.

\section{MÉTODO}

Este estudo transversal faz parte de um estudo longitudinal populacional e domiciliar, realizado com pessoas idosas (60 anos ou mais), residentes na área urbana de Florianópolis, Estado de Santa Catarina, Sul do Brasil. Os dados deste estudo são oriundos da pesquisa 'Condições de Saúde de Idosos de Florianópolis, denominada EpiFloripa Idoso ${ }^{11,12}$. Segundo o censo demográfico de 2010, o município possuía população total de 421.239 habitantes, sendo as pessoas idosas correspondentes a 11,4\% desse valor. $\mathrm{O}$ índice de desenvolvimento humano municipal (IDHM) era de 0,847 nesse mesmo ano, considerado elevado, ocupando o terceiro lugar entre os municípios e o primeiro dentre as capitais brasileiras $^{13,14}$.

A seleção da amostra da linha de base ocorreu em duas etapas por conglomerado. A primeira unidade foram os setores censitários. Dos 420 setores urbanos de Florianópolis, 80 foram sorteados sistematicamente, o que correspondeu a oito setores em cada decil de renda ( $\mathrm{R} \$ 192,80$ - R $\$ 13.209,50)$. As unidades da segunda etapa foram os domicílios. Foi necessária uma etapa de atualização do seu número em cada setor (matrículas) e foram registrados apenas os endereços residenciais permanentemente ocupados. O número de domicílios variou de 61 a 725 , e para reduzir o coeficiente de variação, de $52,7 \%$ ( $n=80$ setores) para $35,2 \%$ ( $n=83$ setores), foi realizado um agrupamento de pequenos setores, levando em consideração os localização geográfica e o decil de renda correspondente, e a divisão de áreas muito grandes. Foi estimado que 60 famílias por setor precisariam ser visitadas.

A estimativa do tamanho da amostra foi realizada por meio do programa EpiInfo, versão 6.04 (Centers for Disease Control and Prevention, Atlanta, EUA). Baseou-se na fórmula de cálculo da prevalência e nos parâmetros do tamanho da população (44.460), nível de confiança (95\%), prevalência desconhecida (50\%), erro amostral (4 pontos percentuais), efeito do desenho (estimado em 2), mais $20 \%$ para perdas estimadas e $15 \%$ para estudos de associação. Isso resultou em uma amostra mínima de 1.599 indivíduos. A amostra foi ampliada para 1.911, devido à disponibilidade de recursos, mas foram entrevistadas 1.702 pessoas idosas. Desses respondentes da primeira onda, foram identificadas 376 perdas (22,1\%), que incluíram 217 óbitos, e 129 recusas $(7,6 \%)$, totalizando 1.197 participantes em 2013/2014 (taxa de resposta de 70,3\%).

Os dados foram coletados por meio de entrevistas individuais realizadas por entrevistadores treinados. Foram utilizados instrumentos validados e desenvolvido um estudo piloto $(\mathrm{n}=99$ na primeira onda $n=76$ na segunda). Em 2009/2010 as entrevistas foram gravadas em dispositivos digitais portáteis (PDA) e em 2013/2014 em netbooks.

O controle de qualidade do EpiFloripa Idoso foi realizado por meio da aplicação de um questionário reduzido por telefone (com oito questões) em cerca de $10 \%$ das pessoas idosas da amostra, selecionados aleatoriamente. A reprodutibilidade das questões apresentou concordância satisfatória a boa (primeira onda, kappa entre 0,6 e 0,9; e segunda onda, kappa entre 0,5 e 0,9$)$.

Para o presente estudo foram aplicados, sobre a amostra da segunda onda, os seguintes critérios de inclusão: o idoso, exclusivamente, deve ter respondido na íntegra os questionários de QV (CASP-19) ${ }^{4}$ e VPI 
(CTS-1) ${ }^{14}$ e, possuir parceiro íntimo nos últimos doze meses. Dos 1.197 participantes, 458 não tiveram parceiro íntimo nos últimos 12 meses, 57 entrevistas foram respondidas por informantes, houve 31 recusas de resposta ao CTS-1 e 2 entrevistas incompletas do CASP-19, totalizando 649 pessoas idosas elegíveis, com taxa de resposta de 54,2\%. O fluxograma da amostra é apresentado na Figura 1.

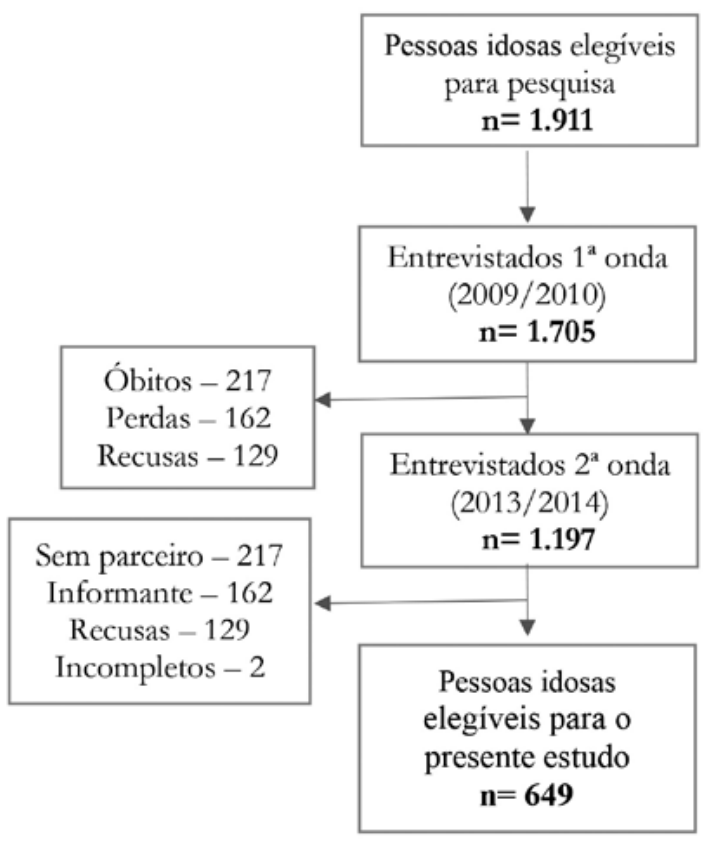

Figura 1. Fluxograma do tamanho da amostra de estudo. Estudo Epi Floripa. Florianópolis, SC, 2013/2014.

O desfecho deste estudo, qualidade de vida (QV), foi mensurado pelo instrumento CASP-19. Essa escala compreende quatro domínios, distribuídos em 19 itens: controle (idade é uma limitação; não há controle sobre o que acontece consigo; sentese livre para planejar o futuro; sente-se excluído de tudo), autonomia (pode fazer o que quer; as responsabilidades familiares impedem de fazer o que deseja; sente-se livre para fazer as coisas; condições de saúde e falta de dinheiro são limitações), realização pessoal (sente-se animado; percebe que a vida tem sentido; gosta do que faz; gosto da companhia dos outros; sente-se feliz ao olhar para o passado) e prazer (sente-se cheio de energia; escolhe fazer coisas novas; tem satisfação com o rumo que a vida tomou; sente que a vida está cheia de oportunidades e que o futuro parece bom). Tais domínios possuem o mesmo nível de importância, sem organização hierárquica. Para cada item, há quatro opções de resposta em escala Likert (frequentemente, às vezes, raramente, nunca). Para cada resposta se atribuiu um escore, a pontuação geral do CASP-19 varia de 0 , que representa a ausência completa de QV, a 57 quando há satisfação total ${ }^{4}$.

A QV foi mensurada pelo escore total (CASP19), e seus domínios agrupados em controle e autonomia (escores de 0 a 27 ) e realização pessoal e prazer (escores de 0 a 30). Essa divisão apresenta sustentação teórica na literatura ${ }^{15,16}$ onde se verificou 
que os quatro domínios do CASP-19 não foram suficientemente distintos para sua análise isolada. Análises psicométricas, conduzidas no Leste Europeu $^{17}$ e na Irlanda ${ }^{18}$, concluíram que o controle e autonomia, relativos à capacidade individual de iniciar e alcançar objetivos; a realização pessoal e prazer, que representam o alcance pleno do potencial humano; quando agrupados representam melhor a QV das pessoas idosas.

A variável de exposição foi VPI, mensurada pela adaptação transcultural do instrumento Conflict Tatics Scales Form R (CTS-1) ${ }^{15}$, desenvolvido para aferir violência no casal. O questionário investiga presença de atos de agressão verbal em 6 itens de xingamentos e ameaças (xingou ou insultou; ficou emburrado; retirou-se do local; fez/disse coisas para irritar; ameaçou bater ou jogar coisas; destruiu/ jogou objetos) e agressão física em 9 itens de força física ou explícita (jogar objetos; empurrar/agarrar; dar tapas ou bofetadas; chutar, morder ou dar murro; bater ou tentar bater com objetos; espancar; estrangular/sufocar; ameaçar com faca ou arma). Foi possível verificar a direcionalidade da violência, pois foi perguntado ao entrevistado se ele cometeu o ato contra o parceiro (violência perpetrada) e, se o parceiro cometeu contra ele (violência sofrida). Quando o mesmo indivíduo sofreu e perpetrou violência, classificou-se como violência bidirecional. Considerou-se a presença de VPI, quando a resposta foi positiva pelo menos para um dos itens da escala, no período recordatório referente aos últimos 12 meses. Essa variável foi transformada em variável dummy, assumindo valores iguais a 0 ou 1 estratificando a amostra em sim e não. O CTS-1 foi utilizado em outros estudos brasileiros ${ }^{19,20}$ com boa confiabilidade e baixa taxa de recusa.

As variáveis de ajuste foram: faixa etária (60- 69, 70- 79 e 80 anos ou mais), renda familiar per capita em salários mínimos $(<1,1-5,5-10,>10)$, deficit cognitivo (não ou provável), sintomas depressivos (não ou suspeita à depressão), dependência das atividades de vida diária (AVD) categorizadas ausente, leve, moderada/grave. Mensurou-se o deficit cognitivo pelo Mini Exame do Estado Mental de Folstein $(\mathrm{MEEM})^{21}$, a presença de sintomas depressivos foi avaliada pela Escala de Depressão Geriátrica
(GDS-15) ${ }^{22}$ e a dependência em AVD pela Escala das Atividades da Vida Diária de BOMFAQ/OARS ${ }^{23}$.

Inicialmente, realizou-se estatística descritiva das variáveis de ajuste (faixa etária, renda, deficit cognitivo, sintomas depressivos e dependência em AVD) apresentando-se frequências absolutas e relativas, estratificadas por sexo. O nível QV foi apresentado por meio do escore total e pelos domínios que o compõem: controle e autonomia; realização pessoal e prazer. Utilizaram-se medidas de tendência central (média) e dispersão (desviopadrão), segundo as variáveis de ajuste para homens e mulheres. A QV foi expressa em média e intervalos de confiança de 95\% (IC95\%), de acordo com as variáveis independentes. As médias foram comparadas pelos testes $t$ de Student (sexo), MannWhitney (sintomas depressivos e deficit cognitivo) e Kruskal-Wallis (faixa etária, renda e dependência em AVD) e não paramétricos de tendência para as variáveis idade, renda e dependência em AVD.

Procedeu-se a análise de associação entre as variáveis de VPI (sofrida, perpetrada e bidirecional) e os escores de QV e seus domínios agrupados (controle e autonomia; realização pessoal e prazer), por meio da regressão linear múltipla. $\mathrm{Na}$ análise não ajustada e ajustada foram apresentados os resultados em coeficiente beta $(\beta)$ e seus respectivos intervalos de 95\% de confiança (IC95\%). Foram conduzidos três modelos de regressão: escore total de QV; controle e autonomia; realização pessoal e prazer, estratificados por sexo. $\mathrm{Na}$ análise ajustada, cada variável de exposição, referente à VPI (sofrida, perpetrada e bidirecional), foi controlada pelas variáveis de ajuste, a fim de estimar seu efeito sobre os escores de QV e domínios. Fixou-se o nível de significância estatística em 5\% para associação.

Nos modelos ajustados de regressão linear procedeu-se à análise dos resíduos, por meio da avaliação da heterocedasticidade e normalidade, da verificação dos resíduos padrão e do fator de inflação da variância (VIF). Para análise dos dados, foi utilizado software estatístico. Considerou-se o efeito do desenho amostral por conglomerados e incorporados os pesos amostrais. 
O estudo EpiFloripa Idoso foi aprovado pelo Comitê de Ética em Pesquisa com Seres Humanos da Universidade Federal de Santa Catarina (protocolo 352/2008 e CAAE 16731313.0.0000.0121), todos os participantes assinaram termos de consentimento livre e esclarecido.

\section{RESULTADOS}

Das 1.197 das pessoas idosas da segunda onda, 649 compuseram o estudo, com taxa de resposta de $54,2 \%$. Verificou-se que os participantes do estudo $(n=649)$ apresentaram maiores escores de QV $(46,3)$ quando comparados aos não participantes $(44,9)$, com significância estatística $(p=0,008)$. Os não participantes $(n=548)$ em maioria eram do sexo feminino (85,2\%), com mais de 70 anos (76,4\%), escolaridade inferior a 4 anos de estudo e renda inferior a cinco salários mínimos, bem como com maiores prevalências de depressão, deficit cognitivo e dependência em AVD, quando comparados aos participantes.

A análise descritiva da amostra e escores médios de QV e seus domínios, segundo condições demográficas, socioeconômicas e de saúde em homens e mulheres são descritos na Tabela 1.

A maioria dos participantes foram homens (52,4\%), destes 45,1\% com idade entre 70-79 anos, $58,6 \%$ possuem renda familiar acima de cinco salários mínimos e 43,2\% não apresentam dependência em AVD. Entre as mulheres, predominou a faixa etária de 60-69 anos (47,2\%), renda familiar de 1 a 5 salários mínimos $(50,5 \%)$ e dependência em AVD leve (47,1\%). Para ambos os sexos, houve ausência de sintomas depressivos e de provável deficit cognitivo na maioria dos indivíduos.

A média dos escores de QV mensurados foi de 46,8 (DP=7,5) entre homens e 45,8 ( $\mathrm{DP}=8,1)$ entre mulheres $(p=0,093)$. As mulheres tiveram escores significativamente inferiores $(20,5 ; \mathrm{DP}=5,0)$ de controle e autonomia quando comparadas aos homens $(21,3 ; \mathrm{DP}=4,6)(p=0,042)$. No domínio de realização pessoal e prazer as médias foram de $25,5(\mathrm{DP}=4,2)$ para homens e 25,2 $(\mathrm{DP}=4,2)$ para mulheres $(p=0,405)$, sem diferença estatisticamente significativa entre os sexos.
A QV geral foi maior segundo a renda apenas para mulheres $(p=0,021)$. Em homens e mulheres, os níveis de realização pessoal/ prazer foram maiores segundo aumento da renda e menores de acordo com aumento da idade, apresentando tendência linear. $\mathrm{Na}$ presença de sintomas depressivos, deficit cognitivo e dependência em AVD, verificaram-se menores escores de QV e seus domínios, exceto para controle/ autonomia nas mulheres.

Ao mensurar as médias dos escores de QV, mulheres em situação de VPI nas três direções analisadas (sofrida, perpetrada e bidirecional) mostraram menores níveis de QV. Mulheres que perpetraram VPI tiveram menores escores de QV (-3,15; IC95\%: -4,84; -1,45), seguidas pelas envolvidas na VPI bidirecional $(-2,59$; IC95\%: -4,10; -1,09) e pelas que sofreram violência (-1,62; IC95\%: -3,06; $-0,17)$ na análise ajustada (Tabela 2). Demonstrase o comprometimento da QV quando a mulher se envolve em situação de VPI, principalmente quando são responsáveis pelos atos de agressão.

Ao analisar os domínios de QV após ajustes, os escores de controle e autonomia foram menores entre as mulheres que perpetraram violência $(-1,62$; IC95\%: $-2,70 ;-0,55)$ e para aquelas em situação de violência bidirecional (-1,36; IC95\%: -2,41; -0,31) (Tabela 3). Quanto ao domínio de realização pessoal e prazer, os escores foram menores para mulheres perpetradoras de violência (-1,52; IC95\%: -2,50; -0,54), seguida pelas envolvidas na violência bidirecional (-1,23; IC95\%: $-2,12 ;-0,34)$ e para as que sofreram VPI $(-0,81$; IC95\%: -1,58; -0,04), na análise ajustada (Tabela 4).

Nos homens, as análises não ajustadas e ajustadas demostraram que não houve diferenças sobre o escore de QV e seus domínios, entre aqueles em situação de VPI nas três direcionalidades analisadas comparados aos não expostos à violência. Ressalta-se que vivenciar a VPI produziu efeitos negativos apenas sobre a QV das mulheres, enquanto os homens não foram afetados.

$\mathrm{Na}$ análise de resíduos, verificou-se que esses apresentaram distribuição normal, não houve erros de especificação nos modelos de regressão, segundo a análise de resíduos padrão, e não se identificou multicolinearidade usando o fator de inflação da variância (VIF). 
Tabela 1. Análise descritiva e escores de qualidade de vida estratificadas por sexo segundo condições demográficas, socioeconômicas e condições de saúde em mulheres e homens. EpiFloripa Idoso, Florianópolis, Santa Catarina, 2013/2014.

\begin{tabular}{|c|c|c|c|c|c|c|c|c|}
\hline & \multirow{3}{*}{$\begin{array}{l}\text { Homens } \\
\mathrm{n}(\%)\end{array}$} & \multirow{3}{*}{$\begin{array}{c}\text { Mulheres } \\
\mathrm{n}(\%)\end{array}$} & \multicolumn{2}{|c|}{ CASP-19 } & \multicolumn{2}{|c|}{$\begin{array}{l}\text { Controle e } \\
\text { Autonomia }\end{array}$} & \multicolumn{2}{|c|}{$\begin{array}{c}\text { Realização pessoal e } \\
\text { Prazer }\end{array}$} \\
\hline & & & Homens & Mulheres & Homens & Mulheres & Homens & Mulheres \\
\hline & & & $\begin{array}{l}\text { Média } \\
(\mathrm{DP})\end{array}$ & $\begin{array}{l}\text { Média } \\
\text { (DP) }\end{array}$ & $\begin{array}{l}\text { Média } \\
(\mathrm{DP})\end{array}$ & $\begin{array}{l}\text { Média } \\
(\mathrm{DP})\end{array}$ & $\begin{array}{l}\text { Média } \\
(\mathrm{DP})\end{array}$ & $\begin{array}{l}\text { Média } \\
(\mathrm{DP})\end{array}$ \\
\hline & $340(52,4)$ & $309(47,6)$ & $46,8(7,5)$ & $45.8(8,1)$ & $21,3(4,6)$ & $20,5(5,0)$ & $25,5(4,2)$ & $25,2(4,2)$ \\
\hline$p^{* * *}$ & & & 0,0 & 93 & 0,0 & 42 & & 05 \\
\hline Faixa etária (anos) & $\mathrm{n}=340$ & $\mathrm{n}=309$ & & & & & & \\
\hline $60-69$ & $135(37,5)$ & $147(47,2)$ & $47,9(6,9)$ & $46,6(7,8)$ & $21,9(4,2)$ & $20,8(5,0)$ & $26,0(4,3)$ & $25,8(4,0)$ \\
\hline $70-79$ & $146(45,1)$ & $129(42,0)$ & $46,5(7,4)$ & $44,8(8,4)$ & $21,2(4,6)$ & $20,1(5,3)$ & $25,3(4,0)$ & $24,7(4,3)$ \\
\hline 80 ou mais & $59(17,4)$ & $33(10,8)$ & $44,9(8,7)$ & $45,6(7,7)$ & $20,1(5,1)$ & $21,1(4,7)$ & $24,7(4,4)$ & $24,4(4,5)$ \\
\hline$p^{*}$ & & & 0,053 & 0,171 & 0,084 & 0,491 & $0,030^{\#}$ & $0,016^{\#}$ \\
\hline Renda (salário mínimo) & $\mathrm{n}=329$ & $\mathrm{n}=288$ & & & & & & \\
\hline Menor que 1 & $9(3,3)$ & $15(4,2)$ & $45,4(7,2)$ & $43,1(8,6)$ & $21,2(3,7)$ & $19,0(5,4)$ & $24,1(4,5)$ & $24,0(4,0)$ \\
\hline Entre 1- 5 & $138(38,1)$ & $142(50,5)$ & $45,8(8,6)$ & $44,9(8,5)$ & $21,0(5,0)$ & $20,1(5,4)$ & $24,8(4,8)$ & $24,8(4,6)$ \\
\hline Entre 5- 10 & $77(24,8)$ & $76(26,3)$ & $46,7(6,4)$ & $45,9(7,3)$ & $21,4(4,4)$ & $20,9(4,2)$ & $25,2(3,5)$ & $25,0(4,0)$ \\
\hline Maior que 10 & $105(33,8)$ & $55(19,0)$ & $48,0(6,8)$ & $48,5(6,7)$ & $21,5(4,2)$ & $21,6(4,9)$ & $26,4(3,8)$ & $26,9(2,9)$ \\
\hline$p^{*}$ & & & 0,242 & $0,021^{\#}$ & 0,933 & 0,166 & $0,003^{\#}$ & $0,003^{\#}$ \\
\hline Deficit Cognitivo & $\mathrm{n}=340$ & $\mathrm{n}=307$ & & & & & & \\
\hline Não & $290(87,3)$ & $243(80,0)$ & $47,4(6,9)$ & $46,3(7,8)$ & $21,6(4,2)$ & $20,8(5,0)$ & $25,7(3,9)$ & $25,5(4,1)$ \\
\hline Provável & $50(12,7)$ & $65(20,0)$ & $43,3(9,7)$ & $43,7(8,8)$ & $19,3(5,9)$ & $19,5(5,4)$ & $24,0(5,2)$ & $24,2(4,5)$ \\
\hline$p^{* *}$ & & & 0,005 & 0,021 & 0,016 & 0,075 & 0,021 & 0,033 \\
\hline Sintomas Depressivos & $\mathrm{n}=340$ & $n=308$ & & & & & & \\
\hline Não & $290(85,7)$ & $243(80,8)$ & $48,5(5,9)$ & $48,1(6,7)$ & $22,0(4,2)$ & $21,9(4,3)$ & $26,4(3,0)$ & $26,2(3,5)$ \\
\hline Suspeita à depressão & $50(14,3)$ & $65(19,2)$ & $36,6(8,3)$ & $37,1(7,2)$ & $16,7(4,4)$ & $15,5(4,5)$ & $19,8(5,9)$ & $21,7(4,9)$ \\
\hline$p^{* *}$ & & & $<0,001$ & $<0,001$ & $<0,001$ & $<0,001$ & $<0,001$ & $<0,001$ \\
\hline $\begin{array}{l}\text { Dependência em } \\
\text { atividades da vida diária }\end{array}$ & $\mathrm{n}=340$ & $\mathrm{n}=309$ & & & & & & \\
\hline Ausência & $146(43,2)$ & $83(26,7)$ & $51,0(4,1)$ & $50,6(5,5)$ & $23,6(3,0)$ & $23,5(3,8)$ & $27,3(2,2)$ & $27,1(2,6)$ \\
\hline Leve & $135(39,6)$ & $138(47,1)$ & $45,4(7,6)$ & $46,4(7,4)$ & $20,7(4,3)$ & $21,0(4,3)$ & $24,7(4,6)$ & $25,4(4,4)$ \\
\hline Moderada/ Grave & $59(17,2)$ & $88(26,2)$ & $39,6(7,5)$ & $40,2(8,0)$ & $16,8(4,6)$ & $17,1(5,2)$ & $22,7(5,1)$ & $23,1(4,3)$ \\
\hline$p^{*}$ & & & $<0,001^{\#}$ & $<0,001^{\#}$ & $<0,001^{\#}$ & $<0,001^{\#}$ & $<0,001^{\#}$ & $<0,001^{\#}$ \\
\hline
\end{tabular}

* teste Kruskal- Wallis; ** teste Mann- Whitney; ${ }^{* * *}$ teste T de Student, comparação de médias de qualidade de vida e domínios, em homens e mulheres; ${ }^{*}$ tendência, de acordo com teste de tendência não paramétrico para grupos ordinais. 
Tabela 2. Análise não ajustada e ajustada do escore total de qualidade de vida (CASP-19) segundo violência por parceiro íntimo sofrida, perpetrada e bidirecional, estratificada por sexo. EpiFloripa Idoso, Florianópolis, Santa Catarina. 2013/2014.

\begin{tabular}{|c|c|c|c|c|c|c|c|c|}
\hline & \multicolumn{4}{|c|}{ Análise não ajustada } & \multicolumn{4}{|c|}{ Análise ajustada* } \\
\hline & \multicolumn{8}{|c|}{ Qualidade de Vida - CASP 19} \\
\hline & \multicolumn{2}{|c|}{ Homens } & \multicolumn{2}{|c|}{ Mulheres } & \multicolumn{2}{|c|}{ Homens } & \multicolumn{2}{|c|}{ Mulheres } \\
\hline & $\beta(\mathrm{IC} 95 \%)$ & $p$ & $\beta(\mathrm{IC} 95 \%)$ & $p$ & $\beta(\mathrm{IC} 95 \%)$ & $p$ & $\beta(\mathrm{IC} 95 \%)$ & $p$ \\
\hline \multicolumn{9}{|c|}{ Violência sofrida } \\
\hline Não & 1 & & 1 & & 1 & & 1 & \\
\hline Sim & $\begin{array}{c}0,14 \\
(-2,16 ; 2,45)\end{array}$ & 0,901 & $\begin{array}{c}-2,37 \\
(-4,37 ; 0,37)\end{array}$ & 0,022 & $\begin{array}{c}0,31 \\
(-1,24 ; 1,86)\end{array}$ & 0,691 & $\begin{array}{c}-1,62 \\
(-3,06 ; 0,17)\end{array}$ & 0,021 \\
\hline \multicolumn{9}{|c|}{$\begin{array}{l}\text { Violência } \\
\text { perpetrada }\end{array}$} \\
\hline Não & 1 & & 1 & & 1 & & 1 & \\
\hline Sim & $\begin{array}{c}-0,73 \\
(2,33 ; 0,86)\end{array}$ & 0,364 & $\begin{array}{c}-3,90 \\
(-5,66 ; 2,15)\end{array}$ & $<0,001$ & $\begin{array}{c}-0,08 \\
(-1,38 ; 1,21)\end{array}$ & 0,900 & $\begin{array}{c}-3,15 \\
(4,84 ;-1,45)\end{array}$ & $<0,001$ \\
\hline \multicolumn{9}{|c|}{$\begin{array}{l}\text { Violência } \\
\text { bidirecional }\end{array}$} \\
\hline Não & 1 & & 1 & & 1 & & 1 & \\
\hline $\operatorname{Sim}$ & $\begin{array}{c}0,35 \\
(-1,90 ; 2,62)\end{array}$ & 0,751 & $\begin{array}{c}-3,58 \\
(-5,58 ;-1,59)\end{array}$ & 0,001 & $\begin{array}{c}0,31 \\
(-1,27 ; 1,91)\end{array}$ & 0,693 & $\begin{array}{c}-2,59 \\
(-4,10 ;-1,09)\end{array}$ & 0,001 \\
\hline
\end{tabular}

* Modelo ajustado por faixa etária, renda, deficit cognitivo, sintomas depressivos e dependência de atividades de vida diária; IC $95 \%=$ intervalo de confiança de $95 \%$

Tabela 3. Análise não ajustada e ajustada do domínio de qualidade de vida - controle e autonomia - segundo violência por parceiro íntimo sofrida, perpetrada e bidirecional, estratificada por sexo. EpiFloripa Idoso, Florianópolis, Santa Catarina, 2013/2014.

\begin{tabular}{|c|c|c|c|c|c|c|c|c|}
\hline & \multicolumn{4}{|c|}{ Análise não ajustada } & \multicolumn{4}{|c|}{ Análise ajustada* } \\
\hline & \multicolumn{8}{|c|}{ Controle e Autonomia } \\
\hline & \multicolumn{2}{|c|}{ Homens } & \multicolumn{2}{|c|}{ Mulheres } & \multicolumn{2}{|c|}{ Homens } & \multicolumn{2}{|c|}{ Mulheres } \\
\hline & $\beta(\mathrm{IC} 95 \%)$ & $\mathrm{p}$ & $\beta(\mathrm{IC} 95 \%)$ & $\mathrm{p}$ & $\beta(\mathrm{IC} 95 \%)$ & $\mathrm{p}$ & $\beta(\mathrm{IC} 95 \%)$ & $\mathrm{p}$ \\
\hline \multicolumn{9}{|c|}{ Violência sofrida } \\
\hline Não & 1 & & 1 & & 1 & & 1 & \\
\hline $\operatorname{Sim}$ & $\begin{array}{c}0,09 \\
(-1,13 ; 1,33)\end{array}$ & 0,870 & $\begin{array}{c}-1,22(-2,57 \\
0,11)\end{array}$ & 0,073 & $\begin{array}{c}0,20 \\
(-0,66 ; 1,02)\end{array}$ & 0,637 & $\begin{array}{c}-0,80 \\
(-1,89 ; 0,27)\end{array}$ & 0,140 \\
\hline \multicolumn{9}{|c|}{$\begin{array}{l}\text { Violência } \\
\text { perpetrada }\end{array}$} \\
\hline Não & 1 & & 1 & & 1 & & 1 & \\
\hline $\operatorname{Sim}$ & $\begin{array}{c}-0,32 \\
(-1,28 ; 0,62)\end{array}$ & 0,490 & $\begin{array}{c}-2,18 \\
(-3,24 ;-1,11)\end{array}$ & $<0,001$ & $\begin{array}{c}-0,01 \\
(-0,81 ; 0,77)\end{array}$ & 0,963 & $\begin{array}{c}-1,62 \\
(-2,70 ;-0,55)\end{array}$ & 0,003 \\
\hline \multicolumn{9}{|c|}{$\begin{array}{l}\text { Violência } \\
\text { bidirecional }\end{array}$} \\
\hline Não & 1 & & 1 & & 1 & & 1 & \\
\hline Sim & $\begin{array}{c}0,15 \\
(-1,03 ; 1,39)\end{array}$ & 0,801 & $\begin{array}{c}-1,95 \\
(-3,20 ;-0,69)\end{array}$ & 0,002 & $\begin{array}{c}0,07 \\
(-0,83 ; 0,98)\end{array}$ & 0,872 & $\begin{array}{c}-1,36 \\
(-2,41 ;-0,31)\end{array}$ & 0,012 \\
\hline
\end{tabular}

* Modelo ajustado por faixa etária, renda, deficit cognitivo, sintomas depressivos e dependência de atividades de vida diária; IC $95 \%$ = intervalo de confiança de $95 \%$. 
Tabela 4. Análise não ajustada e ajustada do domínio de qualidade de vida - realização pessoal e prazer segundo violência por parceiro íntimo sofrida, perpetrada e bidirecional, estratificada por sexo. EpiFloripa Idoso, Florianópolis, Santa Catarina, 2013/2014.

\begin{tabular}{|c|c|c|c|c|c|c|c|c|}
\hline & \multicolumn{4}{|c|}{ Análise não ajustada } & \multicolumn{4}{|c|}{ Análise ajustada* } \\
\hline & \multicolumn{8}{|c|}{ Realização pessoal e Prazer } \\
\hline & \multicolumn{2}{|c|}{ Homens } & \multicolumn{2}{|c|}{ Mulheres } & \multicolumn{2}{|c|}{ Homens } & \multicolumn{2}{|c|}{ Mulheres } \\
\hline & $\beta(\mathrm{IC} 95 \%)$ & $\mathrm{p}$ & $\beta(\mathrm{IC95 \% )}$ & $\mathrm{p}$ & $\beta(\mathrm{IC} 95 \%)$ & $\mathrm{p}$ & $\beta(\mathrm{IC} 95 \%)$ & $\mathrm{p}$ \\
\hline \multicolumn{9}{|c|}{ Violência sofrida } \\
\hline Não & 1 & & 1 & & 1 & & 1 & \\
\hline $\operatorname{Sim}$ & $\begin{array}{c}0,02 \\
(-1,23 ; 1,28)\end{array}$ & 0,964 & $\begin{array}{c}-1,14 \\
(-2,10 ;-0,18)\end{array}$ & 0,022 & $\begin{array}{c}0,10 \\
(-0,84 ; 1,05)\end{array}$ & 0,821 & $\begin{array}{c}-0,81 \\
(-1,58 ;-0,04)\end{array}$ & 0,035 \\
\hline \multicolumn{9}{|c|}{$\begin{array}{l}\text { Violência } \\
\text { perpetrada }\end{array}$} \\
\hline Não & 1 & & 1 & & 1 & & 1 & \\
\hline Sim & $\begin{array}{c}-0,42 \\
(-1,34 ; 0,50)\end{array}$ & 0,371 & $\begin{array}{c}-1,72 \\
(-2,70 ;-0,74)\end{array}$ & 0,011 & $\begin{array}{c}-0,06 \\
(-0,90 ; 0,77)\end{array}$ & 0,872 & $\begin{array}{c}-1,52 \\
(-2,50 ;-0,54)\end{array}$ & 0,003 \\
\hline \multicolumn{9}{|c|}{$\begin{array}{l}\text { Violência } \\
\text { bidirecional }\end{array}$} \\
\hline Não & 1 & & 1 & & 1 & & 1 & \\
\hline $\operatorname{Sim}$ & $\begin{array}{c}0,18 \\
(-1,01 ; 1,38)\end{array}$ & 0,766 & $\begin{array}{c}-1,63 \\
(-2,68 ;-0,59)\end{array}$ & 0,002 & $\begin{array}{c}0,24 \\
(-0,68 ; 1,17)\end{array}$ & 0,697 & $\begin{array}{c}-1,23 \\
(-2,12 ;-0,34)\end{array}$ & 0,007 \\
\hline
\end{tabular}

* Modelo ajustado por faixa etária, renda, deficit cognitivo, sintomas depressivos e dependência de atividades de vida diária; IC95\% = intervalo de confiança de 95 .

\section{DISCUSSÃO}

O presente estudo é relevante na investigação da associação entre VPI e QV em pessoas idosas no Brasil, analisando a violência sofrida, perpetrada e bidirecional em homens e mulheres. Destacase como principal achado, os impactos distintos produzidos pela exposição à VPI sobre a QV de acordo com o sexo. Apenas mulheres tiveram sua QV prejudicada ao estarem em situações de violência. Menores escores de QV foram identificados entre as mulheres idosas que perpetraram violência contra seus parceiros, seguida por aquelas envolvidas na violência bidirecional e, por último, entre as agredidas por seus parceiros. Enquanto os homens não tiveram sua QV afetada ao perpetrar ou sofrer violência.

As pessoas idosas de Florianópolis apresentaram elevada QV (homens=46,8; mulheres $=45,8$ ) quando comparados a estudos de base populacional conduzidos em outros países ${ }^{16,24}$ que utilizaram o mesmo instrumento de mensuração (CASP-19). Na
Irlanda, a QV em idosos foi inferior $(43,8)$, bem como na Inglaterra $(42,5)$. Os escores médios de QV foram semelhantes entre os sexos no estudo atual, diferindo de outros inquéritos populacionais ${ }^{16,23}$. Tais resultados podem estar relacionados ao fato do presente estudo incluiu apenas pessoas idosas com parceiros, os quais tiveram elevado nível de QV quando comparados aos não participantes. Pessoas idosas casadas ou com companheiro, apresentaram QV superior às solteiras. A presença do parceiro é considerada positiva quando há suporte social e valorização pessoal no relacionamento ${ }^{25}$.

Verificou-se que em situações de violência, as mulheres tiveram sua QV prejudicada, a qual se manteve comprometida mesmo após análise ajustada pelos fatores de faixa etária, renda, deficit cognitivo, depressão e dependência em AVD. Enquanto para os homens a QV permaneceu inalterada, independente destes sofrerem ou cometerem tais atos. Esse achado mostra que o impacto da violência sobre a QV difere significativamente entre mulheres e homens idosos. 
As diferenças de gênero podem ser resultado das disparidades existentes na saúde e das formas distintas pelas quais mulheres e homens idosos respondem e lidam com problemas de saúde e experiências adversas. Em nossa cultura papéis de feminilidade e masculinidade contribuem para conflitos entre casais, e estão frequentemente associados a ocorrência de violência no relacionamento ${ }^{26,27}$. O binômio masculinidade e violência, tradicionalmente é compreendido como se o segundo termo pertencesse ao primeiro. Neste cenário de associação entre ser masculino e ser violento, as relações de gênero são construídas e reproduzidas, legitimando a violência como referência para se distinguir o homem da mulher ${ }^{28}$. Tal construção corrobora com as diferenças do impacto da VPI sobre a QV entre os sexos encontradas neste estudo.

Para as mulheres idosas, tanto cometer como sofrer violência, acarreta menores escores de QV. Destaca-se maior impacto negativo sobre a QV para as mulheres perpetradoras de violência contra seus parceiros (-3,15; IC95\%: -4,84; -1,45) quando comparadas às que sofreram as agressões $(-1,62$; IC95\%: -3,06; -0,17). Parece paradoxal que idosas perpetradoras de VPI tenham sua QV mais comprometida em comparação às que sofrem, uma vez que a literatura ${ }^{26,27}$, as graves consequências da violência relacionam-se às mulheres vítimas. $\mathrm{O}$ sofrimento causado pode servir de catalisador para processos de adoecimento instalados ou predispostos, tais como o comprometimento da saúde física, a dependência em atividades de vida diárias, o deficit cognitivo, e uma maior probabilidade de problemas de saúde mental como ansiedade e depressão, e isolamento social na idade avançada ${ }^{28,29}$ além de autopercepção de saúde mais baixa ${ }^{30}$, que comprometem a QV das pessoas envolvidas na violência.

Ressalta-se que embora a coleta de dados sobre os perpetradores seja um desafio, compreender o papel de fatores, como condição socioeconômica, pois a dependência financeira do perpetrador é uma barreira importante para a busca de ajuda, outros como saúde mental e abuso de substâncias, bem como dependências e interdependências entre perpetradores e vítimas, requer atenção, uma vez que a compreensão dessas características é importante na previsão e prevenção do abuso de idosos ${ }^{27,31}$.
Neste estudo, o protagonismo da mulher nas situações de VPI afeta ambos os domínios de QV - controle e autonomia, realização pessoal e prazer. Ressalta-se que, as mulheres são constantemente responsabilizadas pelo zelo e sucesso dos relacionamentos, estando mais propensas a sentir culpa pelo comportamento violento do parceiro, o que impacta na redução do seu bem-estar. A expectativa de que mulheres devam ser cuidadoras, sensíveis e conciliadoras no âmbito familiar, não se admitindo que assumam postura agressiva e violenta ${ }^{32}$, resulta na sua QV ainda mais comprometida quando assumem o papel de perpetradoras de VPI. Alguns autores ${ }^{33,34}$ descrevem a perpetração de VPI pelas mulheres como uma reação de defesa sobre agressões sofridas previamente. A adoção da violência para resolução dos conflitos conjugais evidencia a falta de outros recursos para mediação dos problemas, indicando a vulnerabilidade no relacionamento. Em consonância com tais resultados, estudo americano ${ }^{27}$ encontrou associação entre baixa autoestima e perpetração dessa violência apenas para mulheres.

Deve-se salientar que a VPI entre pessoas idosas não se caracteriza como fato novo, que emerge nesta faixa etária. Estudo de base populacional realizado com adultos de Florianópolis ${ }^{32}$, constatou que as prevalências de sofrer violência física foram elevadas tanto em homens $(17,5 \%)$ como nas mulheres $(16,1 \%)$, porém com consequências negativas em maiores proporções sobre a saúde das mulheres, evidenciado que a violência nos relacionamentos íntimos e seus efeitos se mantém durante a vida. Estudo longitudinal australiano afirmou que a VPI impactou em menor QV para mulheres ao longo de 16 anos de estudo, perpetuando por três gerações, explicitando que os danos da violência sobre quem a vivencia, são intergeracionais ${ }^{35}$.

Ressalta-se o impacto negativo da VPI sobre o bem-estar das idosas entrevistadas, comprometendo o envelhecimento positivo e saudável, apontando para a necessidade de estratégias para prevenção dessa forma de violência nessa fase da vida. As mulheres em situação de violência são mais suscetíveis a permanecerem socialmente isoladas, afastadas de amigos e familiares, mantendo-se restritas ao ambiente doméstico e ao convívio com o autor de violência ${ }^{31}$. As consequências da VPI são profundas, 
impactando na saúde e felicidade dos envolvidos, estendendo-se e afetando o bem-estar de famílias e até comunidades inteiras?.

Embora a prevalência de VPI em pessoas idosas apresente simetria de gênero ${ }^{33}$, os efeitos produzidos pela exposição a violência sobre a QV são assimétricos, visto que estar em situação de VPI não ocasionou quaisquer impactos sobre a QV dos homens. No entanto, esse impacto sobre a QV das mulheres é permeado por uma iniquidade de gênero que deve ser combatida, visto que atos de agressões, abusos e humilhações no relacionamento são socialmente toleráveis ${ }^{30}$.

A partir da teoria de gênero ${ }^{29}$, a adoção de comportamento violento pelo homem contra a parceira, corresponde a forma correta de atuar, historicamente construída e valorizada, para resolver problemas. A violência praticada pelo parceiro permanece naturalizada e banalizada, fazendo com que muitas vezes sequer seja considerada como tal ${ }^{34}$. Além disso, a violência costuma ser reconhecida pelos homens apenas em situações da vida pública: nas relações urbanas, impessoais e/ ou anônimas. Uma vez que, posturas agressivas e atos de violência no âmbito familiar e conjugal, são tidas como o exercício do papel do chefe de família, permanecendo a violência no relacionamento invisibilizada e negligenciada ${ }^{34}$.

Para a redução da VPI sobre homens e mulheres se faz necessária a desconstrução dos papéis hierárquicos de gênero e a redução dos fatores estruturais que apoiam essas desigualdades, as quais certamente são intervenções de grande valia para o enfrentamento da violência e o alcance do envelhecimento saudável, promovendo a QV entre as pessoas idosas, principalmente para as mulheres, as mais afetadas ${ }^{35,36}$. O reconhecimento das situações de violência no relacionamento íntimo entre pessoas idosas nos serviços de saúde, ainda é uma prática incipiente no contexto brasileiro ${ }^{30}$. Existem serviços de atendimento à pessoa idosa vítima de abuso e maus tratos, porém uma parcela maior dessa população pode estar sofrendo consequências da VPI, nos seus relacionamentos.

Sobre o constructo de QV, adotou-se definição específica para população idosa que transcende às questões relacionadas à saúde, mensurando aspectos de percepção subjetiva sobre a vida. A análise de amostra representativa de base populacional de uma capital do Sul do Brasil, conduzida por instrumentos validados e da alta confiabilidade - CTS-1 e CASP19 - alcançou resultados fidedignos, possibilitando concluir que mulheres tiveram sua QV prejudicada por situação de VPI, e que ser perpetradora causou impacto negativo superior a ser vítima, enquanto para os homens essa forma de violência não alterou os níveis de QV.

Entre as limitações encontradas destaca-se o perfil etário da amostra, que incluiu idosos a partir de 63 anos. Esse grupo difere da população alvo do instrumento, que foi planejado para ser aplicado em indivíduos com 50 anos ou mais. Tal limitação potencialmente implicou uma menor variabilidade nas respostas aos itens, atenuando a magnitude de toda a sorte de parâmetros estimados no presente estudo. Outra limitação refere-se à estratégia de aplicação do CASP-19, a qual se deu face-a-face, e não por autopreenchimento, o que pode afetar as respostas aos itens do instrumento no sentido de superestimar a avaliação da QV que resultou do processo. Esse modo de administração foi adotado no estudo para permitir que mesmo pessoas idosas com baixo nível de escolaridade ou acuidade visual pudessem responder ao questionário da pesquisa.

\section{CONCLUSÃO}

A realização deste estudo abordou a associação da VPI sobre a Qualidade de vida (QV) de mulheres e homens idosos. Encontrou-se impacto assimétrico entre os sexos, sendo apenas as mulheres prejudicadas em sua QV, quando vivenciam situações de violência por parceiro íntimo (VPI), seja sofrida, perpetrada ou bidirecional. Destaca-se que há menores escores de QV nas mulheres idosas que cometeram VPI contra seus parceiros.

A VPI tem impactos psicológicos e de saúde significativos em mulheres mais velhas, muitas vezes exacerbados pela duração da violência. Abuso prolongado e transições sociais essenciais, como filhos saindo de casa, podem levar a sentimentos de desesperança, expectativas de vida não satisfeitas e 
profundo isolamento social, reduzindo a qualidade de vida nessa população.

Ressalta-se a importância de promover políticas que auxiliem na prevenção da VPI nas pessoas idosas, visto que essa população enfrenta barreiras para ter acesso a ajuda, além de ser um tema pouco investigado especialmente em termos de intervenções. A partir da importância da QV para a área de gerontologia e do avanço que o CASP-19 representa - por estar alinhado às novas correntes teóricas sobre envelhecimento - esperase que o presente estudo contribua com a noção de envelhecimento saudável e ativo e oriente as ações voltadas para as populações idosas no Brasil e no mundo.

Este estudo é relevante por investigar temática ainda pouco investigada, abordando além da violência sofrida no relacionamento íntimo, a perpetrada e bidirecional. São escassas as pesquisas que comparam os resultados de vitimização e perpetração da VPI em homens e mulheres. Tal conduta, reduz o viés de análise, visto que não se definem previamente vítimas e agressores, mas sim analisam-se tais comportamentos em ambos os sexos.

\section{Editado por: Maria Helena Rodrigues Galvão}

\section{REFERÊNCIAS}

1. Machado DR, Kimura M, Duarte YADO, Lebrão ML. Violência contra idosos e qualidade de vida relacionada à saúde: estudo populacional no município de São Paulo, Brasil. Ciênc Saúde Colet. 2020;25(3):1119-28.

2. Hackenberg EAM, Sallinen V, Koljonen V, Handolin L. Severe intimate partner violence affecting both young and elderly patients of both sexes. Eur J Trauma Emerg Surg. 2017;43(3):319-27.

3. Castro VCD, Rissardo L K, Carreira L. Violence against the Brazilian elderlies: an analysis of hospitalizations. Rev Bras Enferm. 2018;71(Suppl 2):777-85.

4. Hyde M, Wiggins RD, Higgs P, Blane DB. A measure of quality of life in early old age: the theory, development and properties of a needs satisfaction model (CASP-19). Aging Ment Health. 2003;7(3):186-94.

5. Lima F, Hyde M, Chungkham H, Correia C, Campos AS, Campos M, et al. quality of life amongst older Brazilians: a cross-cultural validation of the CASP-19 into Brazilian-Portuguese. PLoS ONE. 2014;9(4):1-10.

6. Cecchetto F, Oliveira QBM, Njaine K, Minayo MCS. Violências percebidas por homens adolescentes na interação afetivo-sexual em dez cidades brasileiras. Interface (Botucatu). 2016;20(59):853-64.

7. Davies R, Lehman E, Perry A, McCall-Hosenfeld JS, Association of intimate partner violence and healthcare provider-identified obesity. Women Health. 2016;56(5):561-75.

8. Warmling D, Lindner SR, Coelho EBS. Prevalência de violência por parceiro íntimo em idosos e fatores associados: revisão sistemática. Ciênc Saúde Colet. 2017;22(9):3111-25.
9. Ferdos J, Rahman M. Exposure to intimate partner violence and malnutrition among young adult Bangladeshi women: cross-sectional study of a nationally representative sample. Cad Saúde Pública. 2018;34(7):1-10.

10. Coker AL, Follingstad DR, Garcia LS, Bush HM. Intimate partner violence and women's cancer quality of life. Cancer Causes Control. 2017;28(1):23-39.

11. Universidade Federal de Santa Catarina. EpiFloripa: condições de saúde de adultos e idosos de Florianópolis. Florianópolis; 2009 e 2013/2014 [Internet]. São Paulo: UFSC; 2009 [acesso em 04 jun. 2020]. Disponível em: http://www.epifloripa.ufsc.br/

12. Schneider IJC, Confortin SC, Bernardo CO, Carvalho CB, Antes DL, Pereira KG, et al. Estudo de coorte EpiFloripa Idoso: métodos, aspectos operacionais e estratégias de seguimento. Rev Saúde Pública. 2017;51:1-10

13. Programa das Nações Unidas para o Desenvolvimento. Relatório do Desenvolvimento Humano 2010: a Verdadeira Riqueza das Nações: vias para o desenvolvimento humano. Portugal: IPAD; 2010. Edição do $20^{\circ}$ Aniversário.

14. Instituto Brasileiro de Geografia e Estatística. Estimativas populacionais residentes, em $1^{\circ}$ de julho de 2009, segundo os municípios [Internet]. Brasília, DF; 2009 [acesso em 04 jun. 2020]. Disponível em: http://www.ibge.gov.br/home/estatistica/populacao/ estimativa2009/POP2009_DOU.pdf

15. Kim GR, Netuveli G, Blane D, Peasey A, Malyutina $S$, Simonova $G$, et al. Psychometric properties and confirmatory factor analysis of the CASP-19, a measure of quality of life in early old age: the HAPIEE study. Aging Ment Health. 2015;19(7):595-609. 
16. Sexton E, King-Kallimanis BL, Conroy RM, Hickey A. Psychometric evaluation of the CASP-19 quality of life scale in an older Irish cohort. Qual Life Res. 2013;22(9):2549-59.

17. Anacleto AJ, Njaine K, Longo GZ, Boing AF, Peres KG. Prevalência e fatores associados à violência entre parceiros íntimos: um estudo de base populacional em Lages, Santa Catarina, Brasil. Cad Saúde Pública. 2007;25(4):800-8.

18. Reichenheim ME, Moraes CL, Szklo A, Hasselmann MH, Souza ERD, Lozana JDA, et al. The magnitude of intimate partner violence in Brazil: portraits from 15 capital cities and the Federal District. Cad. Saúde Pública. 2006;22(2):425-37.

19. Folstein MF, Folstein SE, McHugh PR. "Mini-mental state". A practical method for grading the cognitive state of patients for clinician. J Psychiatr Res 1975;12(3):189-98.

20. Paradela EM, Lourenço RA, Veras RP. Validação da escala de depressão geriátrica em um ambulatório geral. Rev Saúde Pública. 2005;39(6):918-23.

21. Rosa TE, Benício MH, Latorre MR, Ramos LR. Fatores determinantes da capacidade funcional entre idosos. Rev. Saúde Pública. 2003;37(1):40-8.

22. Netuveli G, Wiggins RD, Hildon Z, Montgomery SM, Blane D. Quality of life at older ages: evidence from the English longitudinal study of aging (wave 1). J Epidemiol Community Health. 2006;60(4):357-63.

23. Tampubolon G. Delineating the third age: joint models of older people's quality of life and attrition in Britain 2002-2010. Aging Ment Health. 2015;19(7):576-83.

24. Bartlett BA, Iverson KM, Mitchell KS. Intimate partner violence and disordered eating among male and female veterans. Psychiatry Res. 2018;260:98-104.

25. Pengpid S, Peltzer K. Lifetime spousal violence victimization and perpetration, physical illness, and health risk behaviours among women in India. Int J Environ Res Public Health. 2018;15(12):1-10.
26. Barros CRS, Schraiber LB. Violência por parceiro íntimo no relato de mulheres e de homens usuários de unidades básicas. Rev Saúde Pública.2017;51:1-10.

27. Roberto KA, Hoyt E. Abuse of Older Women in the United States: A Review of Empirical Research, 20172019. Aggres Violent Behav. 2020:1-10.

28. Ho CS, Wong SY, Chiu MM, Ho R. Global prevalence of elder abuse: A metaanalysis and meta-regression. East Asian Arch Psychiatry. 2017;27(2):43-55.

29. Nunes DP, Brito TRPD, Duarte YADO, Lebrão ML. Cuidadores de idosos e tensão excessiva associada ao cuidado: evidências do Estudo SABE. Rev Bras Epidemiol. 2019;21(Supl.2):1-10.

30. Roberto KA, Hoyt E. Abuse of Older Women in the United States: A Review of Empirical Research, 20172019. Aggress Violent Behav, 2020:1-10.

31. Pathak N, Dhairyawan R, Tariq S. The experience of intimate partner violence among older women: a narrative review. Maturitas 2019;121:63-75.

32. Lindner SR, Coelho EBS, Bolsoni CC, Rojas PF, Boing AF. Prevalência de violência física por parceiro íntimo em homens e mulheres de Florianópolis, Santa Catarina, Brasil: estudo de base populacional. Cad Saúde Pública. 2015;31(4):815-26.

33. Loxton D, Dolja-Gore X, Anderson AE, Townsend $\mathrm{N}$. Intimate partner violence adversely impacts health over 16 years and across generations: a longitudinal cohort study. PloS ONE. 2017;12(6):1-10.

34. Yon Y, Mikton CR, Gassoumis ZD, Wilber KH. Elder abuse prevalence in community settings: a systematic review and meta-analysis. Lancet Glob Health. 2017;5(2):147-56.

35. Policastro C, Finn MA. Coercive control and physical violence in older adults: Analysis using data from the National Elder Mistreatment Study. J Interpers Violence. 2017;32(3):311-30.

36. Pathak N, Dhairyawan R, Tariq S. The experience of intimate partner violence among older women: A narrative review. Maturitas. 2019;121:1-10. 